\title{
Distinct as Snowflakes: the Shapes of Silicon Nanoscale Particles
}

\author{
Christopher R. Perrey, Markus Lentzen,* and C. Barry Carter
}

Department of Chemical Engineering and Materials Science, University of Minnesota

421 Washington Avenue S.E., Minneapolis, MN 55455-0132

* Institute for Solid State Research, Research Center Jülich GmbH, 52425 Jülich, Germany

Silicon has immeasurable importance to society as the primary material for electronic applications [1]. As device features continue to reduce in dimension, the properties of silicon nanoparticles have become of great interest. Surface oxide films and defect behavior in these nanoparticles can create either opportunities or problems in device applications. Numerous methods of producing nanoscale $\mathrm{Si}$ have been proposed and demonstrated [2-4]. These routes to the generation of Si nanoparticles can lead to very different results.

Three different plasma-based processes can lead to particles with distinctly different shapes. Figure 1 shows an example of a single-crystal Si nanosphere produced by hypersonic particle plasma deposition (HPPD) $[2,5]$. Similar Si nanospheres have been found to have hardness values which are greater than bulk Si $[6,7]$. The nanospheres have a thin oxide layer that surrounds the particle, and some contain planar interfaces crossing the particle. A formation process which uses an inductively coupled low pressure radio frequency (RF) plasma for the decomposition of heliumdiluted silane can produce more highly oriented nanoparticles of silicon [4]. Figure 2(a) shows a bright-field (BF) TEM image of a Si nanoparticle that exhibits a hexagonal appearance; the particle in Figure 2(b) appears to be a square. If a similar nanoparticle is imaged using a SEM, the 3-D shape of the nanoparticle can be discerned to be of a cubic form that appears as a square in one direction but a hexagon in another. Figure 3 is such a series of SEM images illustrating this highly oriented behavior as the nanoparticle is imaged at different values of tilt. Finally, through the use of silane diluted with both hydrogen and helium in a capacitively coupled RF plasma discharge, Si nanocrystals can be formed. These nanocrystals are deposited in an amorphous Si film. Figure 4 shows two HRTEM images of Si nanocrystals with and without a stacking fault. The particles can be readily seen, through the use of the aberration corrected Philips CM200 FEG, installed at Jülich, which was operated at very small spherical aberration leading to a low image delocalization $[8,9]$.

The differences in the shapes can be related to the differences in the deposition method. Process parameters such as the cooling rate and gas flow likely have impacts, not only in the shape of the silicon nanocrystals, but also to the existence and types of defects observed. These relations can be inferred directly from cross-process studies and by varying the parameters of individual processes. By understanding these relations, the opportunity exists to tailor the shape and size of the $\mathrm{Si}$ nanoparticles to the desired application and to ensure that, unlike real snowflakes $[10,11]$, the shapes of these particles are reproducible.

References

[1] J. Chelikowsky, Mater. Res. Bull., 27 (2002) 951.

[2] N. P. Rao et al., NanoStruct. Mater., 9 (1997) 129.

[3] S. Thompson et al., Bull. Am. Phys. Soc., 47 (2002) 25.

[4] A. Bapat et al., Mater. Res. Soc. Symp. Proc., 740 (2002), in press.

[5] F. DiFonzo et al., Appl. Phys. Lett., 77 (2000) 910.

[6] C. R. Perrey et al., Mater. Res. Soc. Symp. Proc., 740 (2002), in press.

[7] W. W. Gerberich et al., J. Mech. Phys. Solids, (2003), in press.

[8] M. Haider et al., Nature, 392 (1998) 768.

[9] M. Haider et al., Ultramicroscopy, 75 (1998) 53. 
[10] W. A. Bentley and W. J. Humphreys, Snow Crystals, McGraw Hill, New York, 1931.

[11] W. A. Bentley, Snowflakes in Photographs, Dover, New York, 2000.

[12] This work is supported by the National Science Foundation under grant DMI-0103169. The authors thank Prof. Knut Urban, Research Center Jülich, for providing the HRTEM. The project on aberration-correction of a transmission electron microscope was funded by the Volkswagen Stiftung. The University of Minnesota's High Temperature \& Plasma Laboratory provided the material examined.

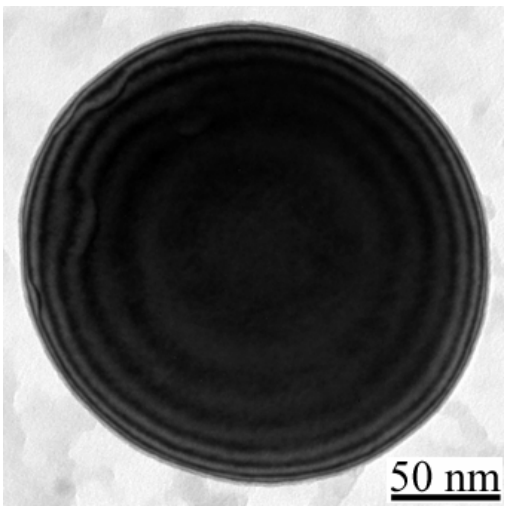

FIG. 1. BF TEM image of a Si nanosphere.
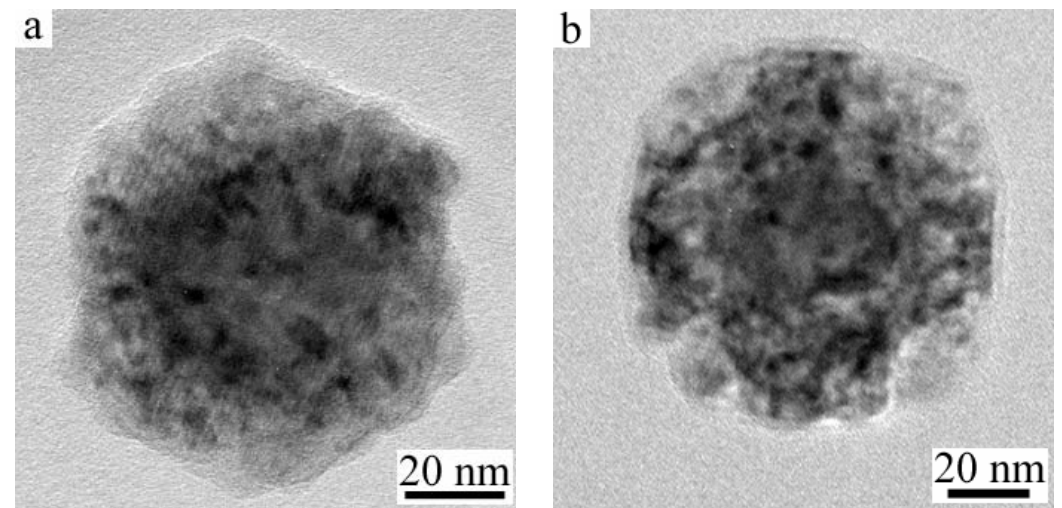

FIG. 2. BF TEM images of different Si nanoparticles (a) hexagonal, (b) square.


FIG. 3. SEM images of a highly oriented Si nanoparticle at different degrees of tilt (rotation axis is noted in (a)). In (a) the particle appears square; in (d) the top half of the particle appears hexagonal.
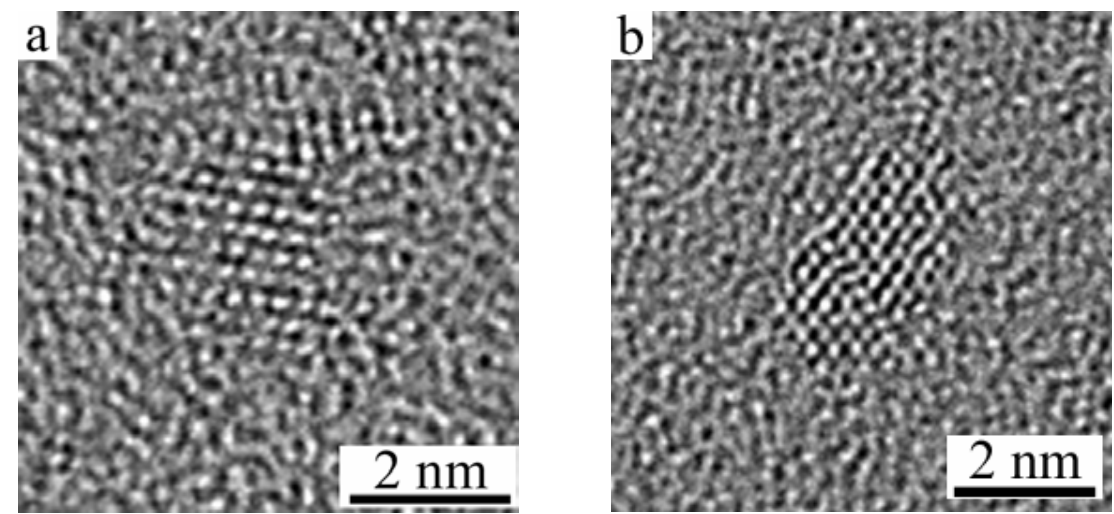

FIG. 4. HRTEM images of nanocrystalline $\mathrm{Si}$ in an amorphous $\mathrm{Si}$ matrix. A single stacking fault is present in (b). 